\title{
Nasopalpebral Schwannomas and Human Immunodeficiency Virus Infection
}

\author{
Adriana Handra-Luca ${ }^{a}$ Djamel Haddar ${ }^{b}$ Anne-Sophie Morin ${ }^{c}$ \\ a Service d'Anatomie Pathologique, Assistance Publique - Hopitaux de Paris (AP-HP), Université Paris Nord \\ Sorbonne Cité, Bobigny, ${ }^{\mathrm{b}}$ Service de Radiologie, and ${ }^{\mathrm{C}}$ Médecine Interne AP-HP, Bondy, France
}

\section{Key Words}

Palpebral area $\cdot$ Nose $\cdot$ Schwannoma $\cdot$ Human

immunodeficiency virus . WT1

\begin{abstract}
Objective: To report a case of a schwannoma of nasopalpebral location, occurring in a human immunodeficiency virus (HIV)-positive patient. Clinical Presentation and Intervention: A 55-year-old man presented with a nasopalpebral painless tumefaction, pneumopathy and HIV-related immunodepression after stopping combination antiretroviral therapy. Magnetic resonance imaging showed subcutaneous masses, with contrast enhancement of the left nose pyramid, internal cantus and inferior palpebral area, suspicious of Kaposi sarcoma. The resected specimen showed schwannoma histology, with tumor cells expressing S100 protein and WT1. Conclusion: The features of a rare case of facial schwannoma of nasopalpebral location in an HIV-positive patient are reported. The diagnosis may be difficult before microscopic examination, with imaging features suggesting a Kaposi sarcoma.

(c) 2015 S. Karger AG, Basel
\end{abstract}

\section{Introduction}

Schwannomas may occur in human immunodeficiency virus (HIV)-positive patients, although rarely. Two cases have been reported to the best our knowledge: one located in the retromandibular upper neck occurred in a 38-year-old woman (without AIDS treatment) and the other occurred in a child $[1,2]$. Bunn and van Heerden [3] in their review of 2012 did not encounter any such case in the head and neck areas. Here we report a case in which a facial schwannoma of palpebral and nasal location occurred in an HIV-positive patient.

\section{Case Report}

A 55-year-old man presented with bilateral pneumopathy that occurred 6 months after he stopped combination antiretroviral therapy for HIV infection (diagnosed 4 years before). The patient showed immunodepression (CD4 lymphocytes at $73 \mathrm{cells} / \mu \mathrm{l})$. The patient also showed a nasopalpebral painless tumefaction of recent increase in size after slow growth. The patient's history revealed left facial paralysis due to a stroke (8 years previously), diabetes, overweight, hypercholesterolemia and arterial hypertension (diagnosed 4 years previously). Magnetic resonance imaging showed a subcutaneous mass of the left nose pyramid associated with smaller nodules of the internal cantus and palpebral area (inferior; fig. 1). The lesions were heterogeneous, showing a strong contrast enhancement after gadolinium injection, suggestive of a Kaposi

Adriana Handra-Luca, MD, $\mathrm{PhD}$

Service d'Anatomie Pathologique, AP-HP GHU Avicenne Université Paris Nord Sorbonne Cité, 125, rue de Stalingrad FR-93009 Bobigny (France)

E-Mail adriana.handra-luca@ hotmail.com 
sarcoma. A surgical excision biopsy was done. The palpebral lesion measured $1.2 \mathrm{~cm}$ and the nasal lesion consisted of 2 lobes of 1.5 and $3.0 \mathrm{~cm}$. The histological features (the specimens were analyzed entirely) were those of schwannoma (fig. 2). A capsule surrounded the tumor nodules. Intratumor vessels were numerous. The majority were small-sized and arranged either isolated (with frequent hyaline change) or grouped as small aggregates. Several reactive lymphocytic foci were observed comprising both CD4 and CD8 T lymphocytes. Tumor cells expressed the $S 100$ protein diffusely and cytoplasmic WT1 focally. They did not express smooth-muscle actin, CD34, CD117 or HHV8. Intratumor CD68-positive macrophages were observed in the subendothelial vessel wall and in small sheets. Tumor cells also expressed CD68 focally. Postoperatively, the patient restarted treatment (combination antiretroviral therapy with atazanavir, ritonavir, tenofovir disoproxil/emtricitabine and trimethoprim/co-trimoxazole). Seven months afterwards the CD4 T lymphocytes were at 354 cells $/ \mathrm{mm}^{3}$ and HIV copies were undetectable.

\section{Discussion}

Schwannomas rarely occur in HIV-positive patients $[1,2]$. The clinical consequences of palpebral tumors might be more life threatening than those of the nasal

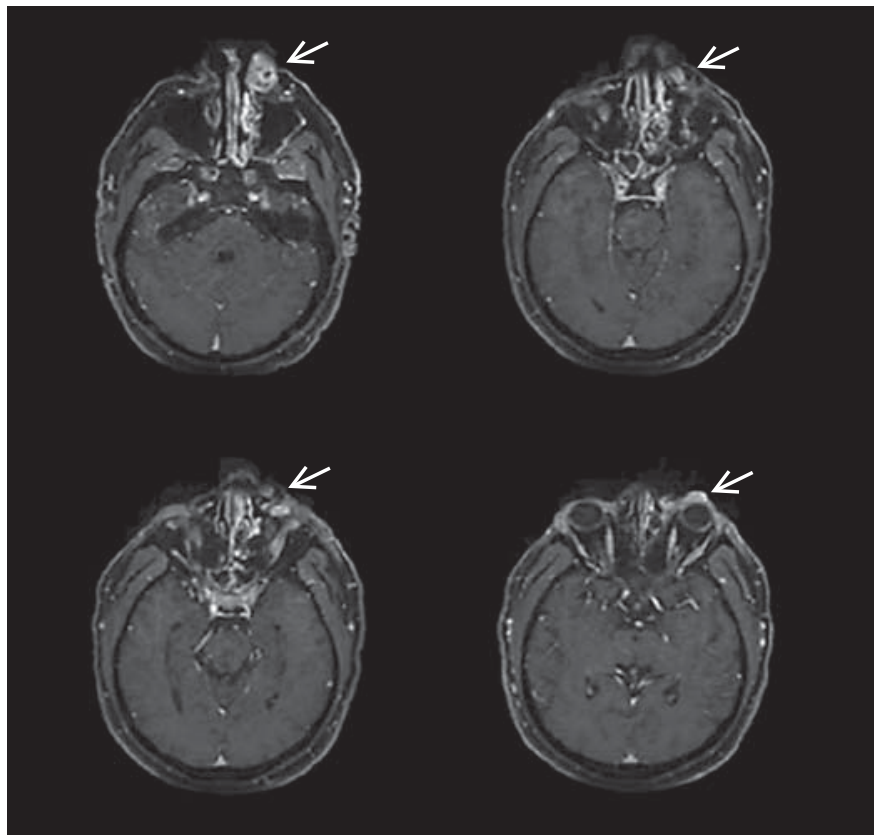

Fig. 1. Axial T1 magnetic resonance imaging (without and with gadolinium injection) showed the nasal and palpebral (inferior) tumors (arrows) with strong contrast enhancement.
Fig. 2. a The tumor consisted of a spindle cell proliferation with Antoni A and B zones. HE stain. b Numerous intratumor vessels and macrophage aggregates were observed between tumor cells or subendothelially (arrows: b HE stain and inset CD68 immunohistochemistry, respectively). The tumor cells expressed S100 and WT1 proteins (immunohistochemistry: c and $\mathbf{d}$, respectively). Intratumor CD4 and CD8 $\mathrm{T}$ lymphocytes were seen in similar proportions (immunohistochemistry: e and $\mathbf{f}$, respectively). Original magnification $\times 10(\mathbf{a}, \mathbf{b}, \mathbf{e}, \mathbf{f}), \times 20(\mathbf{c}, \mathbf{d}), \times 40$ (inset in $\mathbf{b})$.
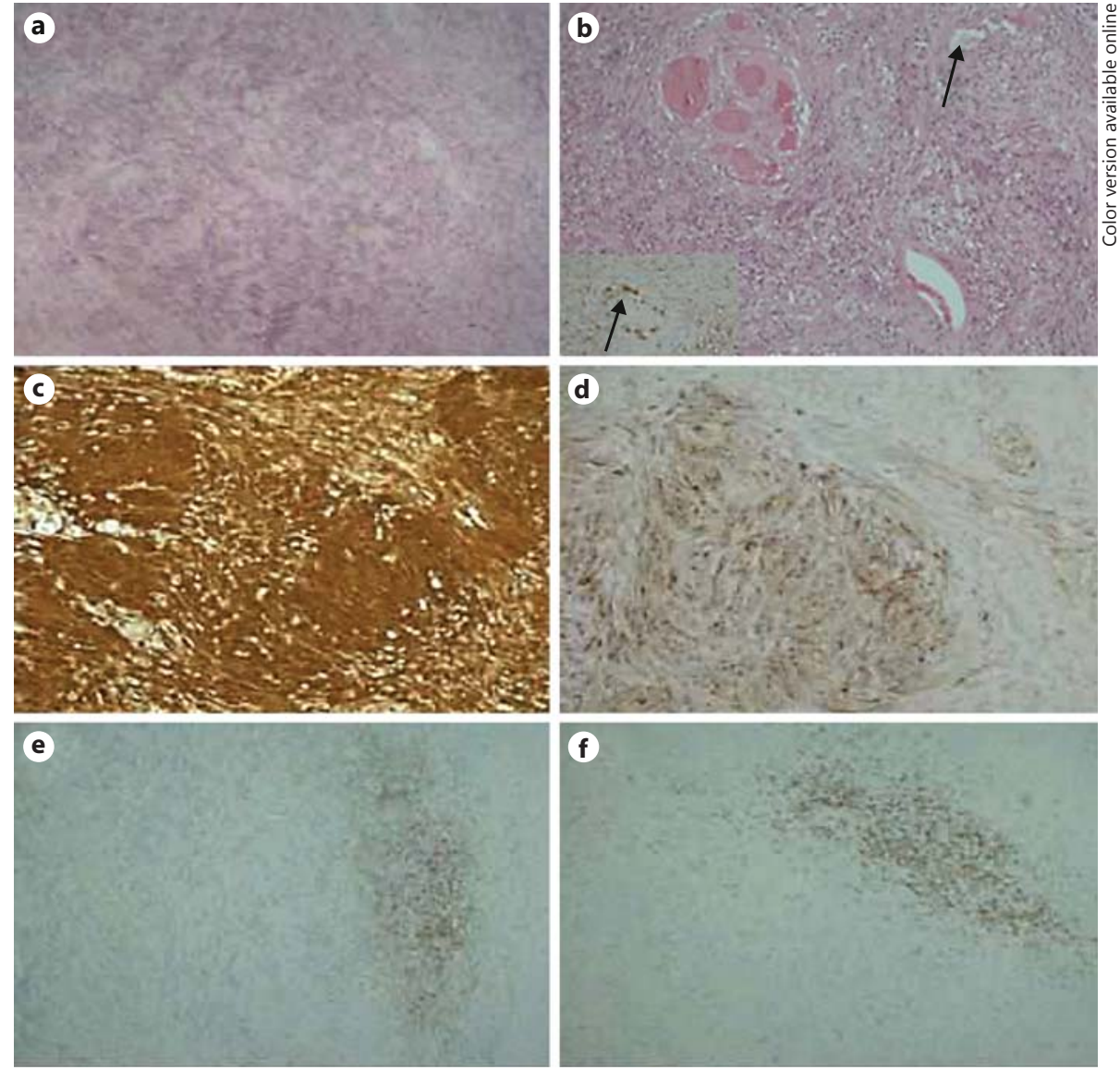
ones. Palpebral schwannomas are rare, with less than 20 cases being reported to date to our knowledge [4]. They are more frequently less than $10 \mathrm{~mm}$ in size [4]. In the case we present, there was no cystic change as previously reported [4] but rare macrophages arranged in sheets or subendothelially. Several factors such as dyslipidemia or injected contrast substance, as noted in the present case, could have contributed to the development of this morphological peculiarity in schwannomas. Although not associated with the lymphocytic reactive foci (tumor-infiltrating lymphocytes), the presence of the macrophages may reflect HIV-related changes. Interestingly, the intratumor reactive lymphocyte infiltrate consisted of quasisimilar numbers of tumor-infiltrating CD4 and CD8 T lymphocytes, although the patient showed low blood CD4 T lymphocytes. This is consistent with previous reports on ovarian serous cystadenocarcinoma, showing no statistically significant differences between intratumor proportions of CD4 and CD8 lymphocytes in HIV-positive and HIV-negative patients, while these differences are statistically significant for serum CD4 and CD8 T lymphocytes [5].

The presurgical diagnosis of schwannomas in HIVpositive patients may be difficult. For instance, in the present case the imaging diagnosis was that of Kaposi sarcoma, a tumor which is more frequent in HIV-positive patients. The cytological diagnosis may be difficult as well, rather suggestive of other more frequent lesions such as granulomas, although rarely biopsied in facial locations [6]. Repeated biopsies and finally surgical exploration may be required [1]. Lack of CD34 expression may be helpful in the differential diagnosis with eyelid solitary fibrous tumors [7]. The CD68 immunohistochemical staining may be misleading since it is positive in both granulomatous and schwannoma cells. Moreover, CD68 and S100 protein expression is observed in eyelid RosaiDorfman disease [8]. Therefore, WT1 immunohistochemical positivity as observed in the present case might be a useful diagnostic tool. Schwannomas express WT1, differentiating them from fibroblastic meningiomas, when intracranial [9]. However, the WT1 involvement in schwannoma pathogenesis in the specific situation of HIV-positive patients might be more complex than that of transcriptional activation suggested for the development of cranial schwannomas. Of note is the observation that INI1 reintroduced to MON and STA-WT1 cells significantly enhance HIV-1 particle production; those produced in STAT-WT1 cells not being reduced for infection as those in MON cells and, INI1 being present in virions produced from STA-WT1 (and not from MON cells) [10].

\section{Conclusion}

The morphological and imaging features of a rare case of facial schwannoma of palpebral and nasal location occurring in a HIV-positive patient were reported. The diagnosis was made only after histological examination, the imaging features suggesting the more frequently encountered Kaposi sarcoma.

\section{Acknowledgments}

The authors would like to thank A. Wassenaar, I. Alexandre, Dr. K.H. Cheng, Prof. J. Schittenhelm and Prof. R. Beschorner. We also thank I. Pluchart, N. Delva, V. Ipotesi, Dr. S. Benzakin, Dr. M. Gerin, Dr. Y. Rabani, M.C. Portenier, the NCA/AP-HP Avicenne, the CMDP/AP-HP and BIUM teams.

\section{References}

1 Pantanowitz L, Kuperman M, Goulart RA: Clinical history of HIV infection may be misleading in cytopathology. Cytojournal 2010; 7:7.

-2 Pollock BH, Jenson HB, Leach CT, et al: Risk factors for pediatric human immunodeficiency virus-related malignancy. JAMA 2003;289: 2393-2399.

>3 Bunn BK, van Heerden WF: HIV/AIDS associated malignancies of the head and neck. SADJ 2012;67:590-592.
4 Cheng KH, Karres J, Kros JM, et al: Cyst-like schwannoma on the eyelid margin. J Craniofac Surg 2012:23:1215-1216.

5 Guidozzi F, Reddy S, Wadee A: Characterization of peripheral blood and ovarian cancerinfiltrating lymphocytes: effect of HIV infection on phenotypic expression and proliferative response. Gynecol Oncol 1994;53:251-255.

-6 Troiano G, Dioguardi M, Giannatempo G, et al: Orofacial granulomatosis: clinical signs of different pathologies. Med Princ Pract 2015; 24:117-122.

7 Pecorella I, Cruciani F, Russo V: A solitary fibrous tumour of the eyelid. Med Princ Pract 2014;23:170-173.
$>8$ Jogai S, Temmim L, Al-Baghli A, et al: RosaiDorfman disease presenting as bilateral eyelid swellings. Med Princ Pract 2006;15:163-165.

$\checkmark 9$ Singh A, Mishra AK, Ylaya K, et al: Wilms tumor-1, claudin-1 and ezrin are useful immunohistochemical markers that help to distinguish schwannoma from fibroblastic meningioma. Pathol Oncol Res 2012;18:383-389.

10 Sorin M, Yung E, Wu X: HIV-1 replication in cell lines harboring INI1/hSNF5 mutations. Retrovirology 2006;3:56 